\title{
IRVING LANGMUIR: O MILAGRE DA CIÊNCIA
}

\section{Marcos Gugliotti}

Departamento de Química Fundamental, Instituto de Química, Universidade de São Paulo, Av. Prof. Lineu Prestes, 748, 05508-900

São Paulo - SP

Recebido em 13/6/00; aceito em 5/12/00

\begin{abstract}
IRVING LANGMUIR: THE MIRACLE OF SCIENCE. Irving Langmuir received the Nobel Prize in Chemistry in 1932 "...for his outstanding discoveries and investigations within the field of surface chemistry”, according to the Swedish Academy. However, few people know that his work comprises other very important contributions, and not only for chemistry, such as the discovery of plasma, the atomic hydrogen, the pure thermoionic phenomenon, the development of the cloud seeding technique for weather modification, among many others. This paper summarizes Langmuir's most important discoveries and theories, with an especial mention for his practical inventions and his work on the atomic theory.
\end{abstract}

Keywords: Irving Langmuir; chemistry; industrial research.

\section{INTRODUÇÃO}

Irving Langmuir ${ }^{1-2}$ nasceu no Brooklyn, Nova Iorque, em 31 de janeiro de 1881 , e foi o terceiro filho de Charles Langmuir, um próspero profissional do ramo de seguros, e Sadie Comings Langmuir. Seus pais viajavam constantemente, em função da carreira de Charles, e as comunicações com os filhos eram feitas por meio de cartas, o que para muitos historiadores fez com que Langmuir desenvolvesse uma notável habilidade em escrever. Langmuir foi muito incentivado por seus pais a observar a natureza, e a manter registros destas observações. Ainda na infância, por influência de seu irmão mais velho Arthur, um químico industrial de prestígio, Langmuir mostrou-se bastante interessado em Ciência. Graduou-se em Engenharia Metalúrgica pela Escola de Minas da Universidade Columbia em 1903, e recebeu o título de Ph.D. em 1906 pela Universidade de Göttingen na Alemanha, sob a orientação de Walther Nernst. A tese de Langmuir foi sobre a dissociação de gases em contato com filamentos aquecidos. De volta aos E.U.A., trabalhou no Instituto de Tecnologia Stevens, em Hoboken, Nova Jersey, como professor do Departamento de Química. Ficou no cargo apenas por três anos, sem oportunidade para pesquisa devido à pesada carga de aulas. Teve um pedido de aumento negado, e publicou um único artigo sem resultados experimentais. Em 1909, durante as férias, conheceu o Dr. Willis R. Whitney, Diretor do Laboratório de Pesquisas da General Electric (GE). Whitney convidou Langmuir para trabalhar durante o verão em seu laboratório, e impressionado por seu talento, ofereceu-lhe um emprego na GE.

Com isso, o mundo acadêmico perdia então a chance de hospedar um futuro ganhador do prêmio Nobel, cujo trabalho pioneiro na indústria é considerado até hoje como sendo o maior exemplo histórico da união Ciência-Tecnologia ${ }^{3-5}$.

\section{O GÊNIO DA LÂMPADA}

Langmuir havia adquirido um bom conhecimento sobre lâmpadas durante seu doutorado, e seus primeiros trabalhos na GE foram motivados por dois fenômenos que ocorriam nas primeiras lâmpadas com filamentos de tungstênio: o escurecimento das paredes internas e o fato de o vácuo melhorar com o

e-mail: bat@quim.iq.usp.br tempo. Com o objetivo inicial de apenas entender esses fenômenos, Langmuir iniciou uma série de experimentos, colocando vários gases em contato com os filamentos. Para poder fazer análises quantitativas numa amostra de gás que ocupava cerca de $1 \mathrm{~mm}^{3}$ sob pressão atmosférica, Langmuir desenvolveu novos tipos de bombas de vácuo. Medindo a resistência dos filamentos de tungstênio, Langmuir estudou a perda de calor em altíssimas temperaturas na presença de vários gases. Percebeu que, no caso do hidrogênio, a perda de calor era 5 vezes maior na temperatura de $3600^{\circ} \mathrm{F}\left(\sim 2000^{\circ} \mathrm{C}\right)$. Porém, quando desligada a corrente elétrica, a pressão no bulbo chegava a zero. Langmuir desconfiou que átomos de hidrogênio estavam sendo formados, e se depositavam na parede interna do bulbo na forma de monocamadas. Confirmou sua hipótese introduzindo oxigênio no bulbo e verificando a estequiometria em função da pressão. A descoberta do hidrogênio atômico levou diretamente à invenção do maçarico de hidrogênio, e despertou a atenção de cientistas como E. Rutherford, G. N. Lewis, e N. Bohr para o trabalho de Langmuir. Continuando seus estudos, percebeu que o único gás que causava escurecimento do bulbo era o vapor de água. Mínimas quantidades produziam um óxido de tungstênio volátil que se depositava nas paredes do bulbo e era reduzido por hidrogênio atômico. O vapor de água produzido por esta redução voltava ao filamento e arrastava mais tungstênio, gerando uma reação em cadeia. Porém, Langmuir observou que ao introduzir outros gases no bulbo, a evaporação de tungstênio era bastante reduzida, e isso poderia aumentar a vida útil das lâmpadas. Enquanto todos queriam fazer vácuo no bulbo, Langmuir queria adicionar mais gás, o que era equivalente a tratar um caso de envenenamento administrando mais veneno. Porém, isso funcionou muito bem com argônio e nitrogênio, e essas lâmpadas foram chamadas de lâmpadas incandescentes preenchidas com gás ("incandescent gas-filled lamps"), a mais famosa patente de Langmuir (U.S. pat. $n^{\circ} 1.180 .159$ ), concedida em 1916. Esta descoberta foi considerada "a segunda revolução em lâmpadas incandescentes", e rendeu uma economia de mais de 1 milhão de dólares por noite na conta elétrica dos americanos (e milhões em faturamento para a GE). Interessado na dinâmica de gases sob baixas pressões em contato com filamentos aquecidos, Langmuir acabou determinando o ponto de fusão do tungstênio, dado valioso para a indústria, e desenvolvendo teorias sobre a transferência de calor em gases. Esses mesmos estudos ainda levaram à descoberta da chamada "carga espacial" 
("space charge") e à comprovação do efeito termoiônico puro. $\mathrm{O}$ trabalho envolvendo descargas elétricas em gases resultou ainda na primeira noção de plasma (termo criado pelo próprio Langmuir ${ }^{*}$ ) como sendo o quarto estado da matéria. Ele ainda projetou melhores tubos de vácuo, utilizados pela indústria do rádio, e também melhores circuitos, levando a GE a ponto de entrar neste mercado e vender o melhor equipamento disponível para o exército dos E.U.A. Desenvolveu tecnologias novas, como melhores bulbos e uma máquina para fazer tungstênio em pasta para a produção de novos filamentos.

Além de melhorar os produtos da GE e trabalhar em várias teorias ao mesmo tempo, Langmuir estava sempre interessado no trabalho dos outros. Uma vez por semana, durante vários anos, costumava ir até uma filial da GE em Massachusetts para ajudar um veterano inventor, Willian Stanley, a desenhar fogões elétricos mais eficientes. O interesse em transferência de calor levou-o também ao estudo dos fornos, resultando em importantes teorias neste campo, como a do fator de forma e em leis de transmissão de calor em máquinas elétricas. Por volta de 1916, Langmuir já era famoso e muito requisitado no circuito científico. Havia recebido seu primeiro grande prêmio, a Medalha Nichols da Sociedade Americana de Química (ACS), e ganhava respeito de mais cientistas como Millikan e Pauli. Influenciado por seus próprios estudos e por palestras de Bragg sobre raios $\mathrm{X}$ e a estrutura de cristais, Langmuir começou a trabalhar em uma nova teoria atômica.

\section{IRVING LANGMUIR E A REGRA DO OCTETO ${ }^{6}$}

O caos pairava sobre as primeiras teorias atômicas, que não explicavam os fenômenos químicos e também não eram bem aceitas pelos físicos. Langmuir já estava interessado no assunto, quando então em 1916, duas publicações importantes aparecem, uma de Kossel e outra de Lewis. Ambas eram similares, baseadas nas hipóteses de Abegg, mas a teoria de Lewis era a mais completa. Lewis imaginava um átomo estático, com os elétrons arranjados em camadas cúbicas em torno do núcleo. A sua teoria, aplicável aos átomos mais leves, era conhecida como "regra dos oito" (nome este dado em função da já conhecida "regra dos dois"). Langmuir refinou e desenvolveu as idéias de Lewis, criando sua própria teoria atômica. $\mathrm{Na}$ teoria de Langmuir, que podia ser aplicada também aos átomos mais pesados e explicava a estabilidade dos gases nobres, os elétrons estavam arranjados em camadas concêntricas em torno do núcleo. Este modelo obteve melhor aceitação tanto pelos químicos como pelos físicos, e foi chamado por Langmuir de "regra do octeto" para não ser confundido com o de Lewis. Em três anos, a partir de 1919, Langmuir publicou doze artigos e apresentou diversas palestras em congressos, as quais acabaram popularizando o modelo de Lewis. Devido à extraordinária capacidade de Langmuir de falar em público e pela semelhança entre os nomes, muitos químicos da época acabavam chamando o modelo de "Lewis-Langmuir theory". No começo, Lewis achou interessante a publicidade, mas com o passar do tempo o crédito pela teoria estava sendo transferido para Langmuir. O modelo começou a ser chamado de "Langmuir-Lewis theory", ou até mesmo "Langmuir theory", o que fez Lewis expressar sua angústia em uma carta ${ }^{6}$ escrita para o Prof. A. A. Noyes em 1926, onde ele disse que "...to persist, especially as they do in England, in speaking of

\footnotetext{
* O termo "plasma" foi criado pelo médico Johannes Purkinje (17971869), para designar "o líquido claro que é obtido quando o sangue é limpo de seus vários corpúsculos". Langmuir fez uma analogia entre um fluido eletrificado que carrega em alta velocidade elétrons, íons, e impurezas e o plasma do sangue, que carrega "corpúsculos vermelhos, brancos e germes". Langmuir chamou um gás ionizado de plasma pela primeira vez em 1927.
}

the Langmuir theory of valence is inexcusable." ("...insistir, especialmente como fazem na Inglaterra, em falar sobre a teoria de valência de Langmuir é indesculpável."). Apesar de aparentemente reconhecer as contribuições de Langmuir para a teoria atômica, Lewis sentiu-se ofuscado pela popularidade que Langmuir havia conseguido, e escreveu em seu famoso livro ${ }^{\dagger}$ o seguinte:

..."This plan, however, was interrupted by the exigencies of war, and in the meantime the task was performed, with far greater success than I could have achieved, by Dr. Irving Langmuir in a brilliant series of some twelve articles, and in a large number of lectures given in this country and abroad. It is largely through these papers and addresses that the theory has received the wide attention of scientists."...

(..."O plano, entretanto, foi interrompido devido à guerra, e durante esse tempo a tarefa foi executada, com um sucesso muito maior que eu poderia ter obtido, pelo Dr. Irving Langmuir numa série de doze artigos, e em um grande número de palestras dadas neste país e no exterior. É em grande parte graças a esses artigos e discursos que a teoria tem recebido grande atenção dos cientistas.....).

..."The theory has been designated in some quarters as the Lewis-Langmuir theory, which would imply some sort of collaboration. As a matter of fact Dr. Langmuir's work has been entirely independent, and such additions as he has made to what was stated or implied in my paper should be credit to him alone."...

(..."A teoria tem sido chamada em alguns lugares como a teoria de Lewis-Langmuir, o que implicaria algum tipo de colaboração. Na verdade, o trabalho do Dr. Langmuir tem sido totalmente independente, e as contribuições que ele tem feito ao que foi explicado ou concluído em meu artigo devem ser creditadas somente a ele."...).

Lewis ainda tentou mostrar a importância de seu trabalho, voltando atrás e dizendo que o modelo da regra dos dois era mais importante que o da regra dos oito. Talvez por pensar que suas idéias estivessem sendo "roubadas" por Langmuir, o que não foi verdade. O trabalho de Lewis foi reconhecido, assim como as importantes contribuições de Langmuir. Linus Pauling, em um artigo de $1984^{8}$, refere-se ao fato da seguinte forma:

..."On the other hand, the two papers that Irving Langmuir published in the Journal of the American Chemical Society in 1919, comprising 84 pages, contained many significant contributions and additions to the theory of the shared electron pair chemical bond. For some reason Lewis was antagonistic to Langmuir, and in my opinion did not give him proper credit."...

("Por outro lado, os dois artigos que Irving Langmuir publicou no Jornal da Sociedade Americana de Química em 1919, num total de 84 páginas, continham várias contribuições e adições significantes à teoria da ligação química por par de elétrons compartilhados. Por alguma razão Lewis foi contra Langmuir, e em minha opinião não deu a ele o devido crédito.”...).

Neste artigo, Pauling menciona alguns dos novos termos $e$ conceitos introduzidos por Langmuir, como eletroneutralidade, união polar (hoje conhecida como ligação iônica), isosterismo, isomorfismo, e ligação covalente, e relembra que Langmuir concluiu ainda que moléculas como $\mathrm{CO}_{2}, \mathrm{~N}_{2} \mathrm{O}$, entre outras, possuíam uma estrutura linear. Foi Langmuir também quem deduziu a expressão matemática para a regra do octeto (a qual é utilizada até hoje), definiu valência, e os termos isoeletrônicos, isômeros e isóbaros.

Mas Langmuir cometeu alguns erros, também citados por Pauling. Ele concluiu que o quartzo continha moléculas de $\mathrm{SiO}_{2}$ com estrutura similar ao $\mathrm{CO}_{2}$, o que não é verdade. Langmuir também achava que a molécula $\mathrm{P}_{4}$ tinha uma estrutura em forma de anel contendo ligações simples e duplas alternadas, não pensando na possibilidade de uma estrutura tetraédrica. 
Porém, não há dúvidas sobre a importância das contribuições de Langmuir para a teoria atômica. Apenas o conteúdo do primeiro artigo de Langmuir sobre o assunto, disse David Harker", "modifica e amplia a teoria da estrutura atômica $e$ molecular de Lewis de tal forma que chega perto de unificar a química em uma só base. Além disso, o modo com o qual Langmuir trata o arranjo dos elétrons nos átomos parece prenunciar os conceitos de química quântica", a qual era recémnascida quando Langmuir enunciou os postulados de sua teoria atômica.

Nos livros didáticos, a teoria (ou regra) do octeto tem como autores Lewis e Kossel. Porém, a contribuição deste último é questionável, o que fica claro nas palavras de Pauling ${ }^{8}$ :

..."Even so, I think that Kossel's paper represented no significant contribution. Much of it is nonsense. He gave a long discussion of electrostatic valence, but nothing about covalence."...

("Mesmo assim, eu acho que o artigo de Kossel não representou uma contribuição significante. A maior parte do artigo não faz sentido. Ele apresentou uma longa discussão sobre valência eletrostática, mas nada sobre covalência.”...).

Diante dos fatos, é natural que naquela época houvesse uma confusão em torno dos nomes dos autores da teoria. Porém, conforme escreveu Albert Rosenfeld ${ }^{1}$, “...Talvez o maior valor da teoria de Lewis - como Joel Hildebrand apontou - foi sua influência no desenvolvimento das idéias de Irving Langmuir. Bem - não importa. Cada cientista reconhece seu débito para com o outro. Vamos chamá-la de Lewis-Langmuir theory".

\section{MÁQUINA DE PENSAR}

Saul Dushman, colega de Langmuir na GE, disse uma vez ${ }^{1}$ : "Langmuir is a regular thinking machine. Put in facts, and you get out a theory" ("Langmuir é uma máquina de pensar regular. Entra-se com os fatos, e extrai-se uma teoria"). Em pouco tempo Langmuir adquiriu a reputação de um "pensador maratonista" na GE, e apesar de muito distraído, impressionava todos que passavam por seu laboratório. "Watching him...", disse Kenneth Kingdon 1 , “...you would say to yourself: this is what science can be like" ("Observando-o, você diria a si mesmo: isto é o que a ciência pode ser").

Ainda com as lâmpadas, Langmuir estudou a adsorção de gases em filamentos, e depois em substratos não-metálicos. Seus trabalhos descreviam fenômenos de adsorção e absorção devido à ação de forças químicas, o que acabou sendo conhecido como quimiossorção. Ele desenvolveu teorias sobre a orientação de moléculas na fase líquida e em interfaces, com especial atenção para o fenômeno da tensão superficial. Em 1916 e 1917, Langmuir publicou dois artigos monumentais sobre as propriedades dos sólidos e líquidos, apresentando neste último (a base de seu prêmio Nobel) a sua mais famosa invenção, a balança de Langmuir, e em 1920 publicou um artigo sobre o mecanismo de flotação.

A Primeira Guerra Mundial já havia começado, e Langmuir foi convidado a trabalhar em pesquisas militares. Ele ajudou a desenvolver os sonares usados para a localização de submarinos (de fundamental importância para o sucesso de várias missões americanas), e dispositivos eletrônicos para a sinalização debaixo da água. Trabalhou na fixação de nitrogênio em explosivos, e melhorou a precisão de torpedos utilizando ondas sonoras. Este último trabalho despertou um certo interesse em Langmuir, o que o levou a estudar fenômenos sonoros. Ele deduziu algumas equações sobre a propagação do som na água e no ar, e ajudou a melhorar a reprodução de gravações da RCA, no desenvolvimento do som estéreo, e na construção de melhores geofones.

Após a guerra, Langmuir passou a ser considerado como um dos maiores cientistas de sua época, e recebia constantemente medalhas e honras de universidades. Em 1929, tornou-se presidente da ACS, e em 1932 recebeu o prêmio Nobel. Nesta época, sua assistente na GE era Katherine Blodgett, e juntos desenvolveram os filmes de Langmuir-Blodgett, mais uma vez abrindo novos campos de pesquisa, principalmente na área de materiais. Os trabalhos de Langmuir de maior impacto no final da década de 30 foram sobre a utilização das monocamadas para o estudo das proteínas, e nesta mesma época, utilizando raios $\mathrm{X}$, ele estudou a estrutura da molécula de insulina.

Com o começo da Segunda Guerra Mundial, Langmuir foi novamente chamado pelo governo para ajudar nas pesquisas militares. Sabia-se que os alemães estavam preparando bombas de gás, e Langmuir foi solicitado para trabalhar no assunto. Utilizando seu conhecimento sobre absorção e adsorção, ele projetou as máscaras de gás utilizadas na Segunda Guerra. Ele ainda construiu geradores para a produção de cortinas de fumaça, que camuflavam as tropas em terra, dificultando a ação de aviões inimigos. Em 1943, o governo pediu para que Langmuir trabalhasse no problema da eletrificação da fuselagem de aviões durante vôos em tempestade. Langmuir então desenvolveu um dispositivo para degelo das asas e fuselagem dos aviões, resolvendo o problema da interferência na comunicação por rádio e na aerodinâmica. Esses trabalhos também despertaram interesse em Langmuir, que começou a estudar fenômenos atmosféricos.

\section{“RAIN MAKER” (“FAZEDOR DE CHUVA”)}

Junto com Vincent J. Schaefer, seu assistente que o ajudou com os trabalhos para a Segunda Guerra, Langmuir começou a estudar como a chuva e a neve eram formadas nas nuvens. Schaefer descobriu acidentalmente em 1946 que o gelo seco funcionava como um excelente núcleo de condensação, necessário para a formação de chuvas. Langmuir acreditava que isso poderia ser usado para mudar o tempo, ou seja, fazer chover ou não, em função da quantidade de núcleos introduzidos numa nuvem. No final da década de 40, Langmuir dirigiu uma série de experimentos sobre nucleação de nuvens, os quais mostraram que a partir de um estímulo infinitesimal, efeitos colossais eram produzidos nas nuvens. Na mesma época, outro colega da GE, Bernard Vonnegut, descobre que iodeto de prata também funcionava como agente de nucleação, e Langmuir utilizou seus geradores de fumaça projetados durante a Segunda Guerra para novos experimentos com AgI. Em abril de 1950, em testes que faziam parte do Projeto Cirrus', Langmuir fez chover de $7 \mathrm{em} 7$ dias em toda a Costa Oeste dos E.U.A., e chegou a usar esta técnica como um método para a supressão de furacões, obtendo resultados até hoje discutidos. Langmuir recebeu por isso o apelido de "rain maker", e tornou-se o primeiro homem "...a fazer algo sobre o tempo". A técnica de nucleação de nuvens tornou-se então um novo campo de pesquisa em Meteorologia, já tendo sido aplicada com sucesso em mais de 50 países.

\section{VIDA SOCIAL E FILOSOFIA}

Dennis Gabor ${ }^{1}$ descreveu Langmuir como “...o mais harmonioso dos espíritos humanos”, e que “...certamente não pertencia ao nosso tempo". Langmuir tinha uma incrível capacidade de concentração, e foi considerado pelos outros grandes cientistas e inventores de sua época como sendo diferente de todos ${ }^{10}$.

Ao contrário do que se possa pensar, ele não ficava trancado em seu laboratório. Praticava diversos esportes ${ }^{1}$, como hipismo, natação, alpinismo e esqui, tendo sido este último introduzido por ele em Göttingen. Preocupado com causas sociais, Langmuir foi um dos fundadores, nos anos 20, da Associação de Proteção ao Lago George no estado de Nova Iorque, um local de lazer onde além de praticar esportes de inverno, ele fazia experiências sobre o gelo e a ação do vento sobre a superfície da água. Langmuir também organizou o primeiro grupo de escoteiros de Schenectady. 
Muito interessado e ativo na área de política científica, Langmuir escreveu vários artigos e apresentou diversas palestras sobre o assunto. Suas maiores preocupações eram com uma legislação específica para a ciência e com o controle da energia atômica ${ }^{11}$.

Langmuir deu grandes contribuições também para a Filosofia da Ciência, mais uma vez introduzindo novos conceitos. Ele dividiu os fenômenos naturais em duas classes: convergentes $e$ divergentes. De acordo com a idéia de Langmuir, um fenômeno convergente é aquele no qual o comportamento de um sistema como um todo pode ser determinado pela média do comportamento de suas partes integrantes, possibilitando previsões estatísticas (como a previsão do número de veículos que irá passar por uma determinada estrada num feriado). Já um fenômeno divergente é aquele no qual um componente isolado, o que poder ser até mesmo uma transformação quântica, tem seu efeito amplificado, de forma que o comportamento do sistema depende de uma mudança infinitesimal, a qual produz grandes efeitos. No caso de um fenômeno divergente, não há relação causa-efeito, o que impossibilita sua previsão. Um exemplo clássico, com o qual Langmuir trabalhou, é o processo de nucleação de nuvens, onde mínimas quantidades de $\mathrm{AgI}$ produzem efeitos colossais na atmosfera (neste caso, por exemplo, $30 \mathrm{mg}$ de AgI distribuídas em aproximadamente $100 \mathrm{~km}^{3}$ de uma nuvem superresfriada, produzem uma quantidade de calor latente de solidificação devido à formação de gelo, que é igual à quantidade de calor liberada nas explosões das primeiras bombas atômicas - veja a ref. 16 do Apêndice). A descrição de Langmuir de um fenômeno divergente explica por que é difícil prever o tempo. Langmuir ainda introduziu o conceito de "Pathological Science"12 ("Ciência Patológica"), estudando fatos inusitados que ocorrem quando se trabalha com ciência, fazendo os cientistas tirarem conclusões absurdas de seus experimentos, e popularizou o termo "serendipity", , definido por ele como "a arte de lucrar a partir de resultados inesperados".

\section{COMENTÁRIOS FINAIS}

Langmuir aposentou-se na GE em 1950, passando a trabalhar como consultor. Ele ainda era chamado para atuar em diversos casos, como para melhorar a eficiência de mísseis teleguiados, acabar com a neblina em Los Angeles, e aumentar a produção em plantações de algodão no Arizona. Passou os últimos anos viajando pelo mundo com sua esposa até que, em 16 de agosto de 1957, faleceu de repente em função de um ataque cardíaco quando visitava a família de um sobrinho em Massachusetts.

O trabalho de Langmuir contribuiu de forma fundamental para diversas áreas da Química ${ }^{13-15}$, Física ${ }^{16}$, Engenharia Elétrica e Mecânica ${ }^{17}, 18$, Meteorologia9 ${ }^{9}$, e até mesmo para a Oceanografia $^{19}$, sendo que para muitos autores ele deveria ter recebido também o prêmio Nobel de Física.

Seus estudos sobre fenômenos básicos, assim como a maioria de suas descobertas, abriram novos campos de pesquisa como: física de plasmas, nucleação de nuvens, catálise heterogênea, filmes de Langmuir (monocamadas), de LangmuirBlodgett (monocamadas em substratos), entre outros. Langmuir é considerado um pioneiro em pesquisa industrial, e sua carreira na GE abriu caminho para o trabalho de cientistas na indústria, especialmente na área de físico-química. Suas invenções estão entre as que mais ajudaram a melhorar a qualidade de vida da humanidade, e Langmuir é atualmente considerado o "fundador da Físico-Química de Superfícies".

Vários cientistas contribuíram para o entendimento da estrutura atômica ${ }^{6}$, como Mendeleev, Abegg, Thomson, Kossel, Ramsay (com uma contribuição bem menor) e Nernst (por seus trabalhos com íons). Porém, as maiores contribuições foram dadas por Lewis e Langmuir. É, portanto, incompreensível o fato do nome de Langmuir não estar associado ao da regra do octeto nos livros de Química (pouquíssimos livros o citam). A citação mais freqüente refere-se à isoterma de adsorção que leva seu nome.

Langmuir foi ainda presidente da Associação Americana para o Avanço da Ciência (AAAS) em 1942, recebeu um total de 15 honras de universidades, 22 medalhas de sociedades científicas, possuiu 63 patentes e publicou cerca de 200 artigos. Levam seu nome: o Instituto de Pesquisas Atmosféricas no Novo México, E.U.A., uma Faculdade na Universidade Estadual de Nova Iorque, um jornal científico ${ }^{20}$ e um prêmio da Sociedade Americana de Química, e até mesmo o "Monte Langmuir", no Alaska.

Langmuir trabalhava no limite entre e Física e a Química, desenvolvendo teorias elegantes e simples ${ }^{21}$, porém com uma apreciável bagagem matemática. Vale lembrar que a academia sueca lhe concedeu o prêmio "...for his outstanding discoveries and investigations..." (“...por suas extraordinárias descobertas e investigações...").

Albert Rosenfeld, editor de ciência da revista Life nos anos 60 , termina a biografia definitiva ${ }^{1}$ de Langmuir descrevendo-o como "the man who was perhaps as great a scientist as has ever been born in America" ("o homem que foi talvez o maior cientista já nascido na América").

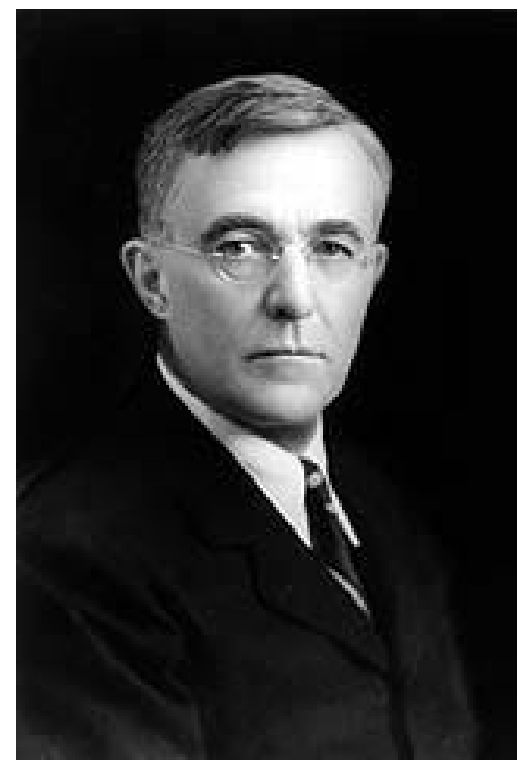

"To-day, however, I propose to tell you of a real two-dimensional world in which phenomena occur that are analogous to those described in 'Flatland"22. I plan to tell you of the behavior of molecules and atoms that are held at the surfaces of three-dimensional solids and liquids."*

* "Hoje, entretanto, eu quero falar sobre um mundo realmente bidimensional no qual os fenômenos que ocorrem são análogos àqueles descritos em 'Flatland'. Eu vou contar-lhes sobre o comportamento de moléculas e átomos que estão presos nas superfícies de sólidos e líquidos tridimensionais."

Irving Langmuir, Science, 84, 379 (1936)

\section{APÊNDICE}

Lista de alguns dos principais artigos de Irving Langmuir relacionados com as teorias e invenções mencionadas no texto.

1- "The Condensation Pump: An Improved Form of High Vacuum Pump", Journal of the Franklin Institute 1916, 182,719

2- "The Dissociation of Hydrogen into Atoms", J. Am. Chem. Soc. 1912, 34, 860

3- "Flames of Atomic Hydrogen", Science 1925, 62, 463

4- "Tungsten Lamps of High-efficiency. I. Blackening of 
Tungsten Lamps and Methods of Preventing it. II. Nitrogen-filled Lamps, (com J. A. Orange), Proc. AIEE 1913, 32, 1894

5- "The Melting Point of Tungsten". Phys. Rev. 1915, 6, 138

6- "Convection and Conduction of Heat in Gases", Phys. Rev. 1912, 34, 401

7- "Flow of Heat Through Furnace Walls: the Shape Factor", (com E. Q. Adams e G. S. Meikle), Trans. Electrochem. Soc. 1913, 24, 53

8- "The Effect of Space Charge and Residual Gases on Thermoionic Currents in High Vacuum", Phys. Rev. 1913, 2, 450

9- "The Pressure Effect and Other Phenomena in Gaseous Discharges", Journal of the Franklin Institute 1923, 196, 751

10- "The Constitution and Fundamental Properties of Solids and Liquids. I. Solids, J. Am. Chem. Soc. 1916, 38, 2221; II. Liquids, J. Am. Chem. Soc. 1917, 39, 1848

11- "The Mechanism of the Surface Phenomena of Flotation", Trans. Faraday Soc. 1920, 15, 62

12- "The Structure of the Insulin Molecule", (com D. M. Wrinch), J. Am. Chem. Soc. 1938, 60, 2247

13- "The Structure of Atoms and the Octet Theory of Valence", Proc. Natl. Acad. Sci. USA 1919, 5, 252

14- "Isomorphism, Isosterism, and Covalence", J. Am. Chem. Soc. 1919, 41, 1543

15- "Types of Valence", Science 1921, 54, 59

16- "Control of Precipitation from Cumulus Clouds by Various Seeding Techniques, Science 1950, 112, 35

\section{REFERÊNCIAS}

1. Rosenfeld, A.; The Quintessence of Irving Langmuir; Pergamon Press, NY, $1^{\text {a }}$ edição, 1966 (O Apêndice desta biografia apresenta a lista completa dos trabalhos de Langmuir. Há um exemplar disponível na Biblioteca do Instituto de Física da USP em São Paulo).

2. Gaines Jr., G. L. In Nobel Laureates in Chemistry, 19011992; James, L. K., Ed.; Washington DC, v. 1, 1993; p 205.

3. Wise, G.; Technology and Culture 1980, 21, 408.

4. Reich, L. S.; Technology and Culture 1983, 24, 199.

5. Wise, G.; Isis 1983, 74, 7.

6. Jensen, W. B.; J. Chem. Educ. 1984, 61, 192.
7. Lewis, G. N. In Valence and the Structure of Atoms and Molecules, The Chemical Catalog Company, Inc., New York, 1923.

8. Pauling, L.; J. Chem. Educ. 1984, 61, 396.

9. Blanchard, D. C.; Bull. Am. Meteorol. Soc. 1996, 77, 1279.

10. Blodgett, K. B.; J. Chem. Educ. 1933, 10, 396.

11. Langmuir, I. In Phenomena, Atoms and Molecules, Philosophical Library, Inc., New York, 1950.

12. Hall, R. N.; "Pathological Science" (Lecture by Irving Langmuir, 1953); Speculations in Science and Technology, 1985, 8, 77-94; Physics Today, Outubro de 1989.

13. Langmuir, A. C.; Industrial and Engineering Chemistry, News Edition 1932, 10, 305.

14. Rideal, R., K.; Nature 1957, 4586, 581.

15. Kerker, M.; J. Coll. Interf. Sci. 1989, 133, 290.

16. Hull, A. W.; Nature 1958, 181, 148.

17. Atherton, W. A.; Electronics world + Wireless world 1990, 96, 605.

18. Brittain, J. E.; Proc. of the IEEE 1995, 83, 1298.

19. Langmuir, I.; Science 1938, 87, 119 (O trabalho de Langmuir nesta área foi sobre a influência do vento na movimentação da superfície da água em mares e lagoas. O fenômeno é conhecido atualmente como "Circulação Langmuir", e além de ter grande importância em biologia marinha, explica também vários casos de "serpentes" que são avistadas nos mares).

20. O jornal "Langmuir", da ACS, teve sua primeira edição em janeiro de 1985. O primeiro editor foi Arthur Adamson, que assina o artigo de inauguração "A Journal is Born" (Langmuir 1985, 1, 1). Karol J. Mysels é quem justifica a escolha do nome em "Why Langmuir", nas páginas seguintes.

21. A principal característica dos trabalhos de Langmuir era a simplicidade. Para os estudos com as lâmpadas, por exemplo, ele utilizou uma instrumentação que consistia basicamente de um balão de vidro, uma bomba de vácuo, um filamento, e um voltímetro.

22. Abbott, Edwin In Flatland: a romance of many dimensions, Smith, P., Ed.; NY, 2a edição, 1992 (Publicado pela primeira vez em 1884, este best seller narra a história de um mundo bidimensional cujos habitantes eram figuras geométricas, e satiriza a hierarquia vitoriana). 